\title{
AN ARITHMETIC PROPERTY OF THE TAYLOR COEFFICIENTS OF ANALYTIC FUNCTIONS WITH AN APPLICATION TO TRANSCENDENTAL NUMBERS
}

\author{
VICHIAN LAOHAKOSOL
}

\begin{abstract}
We extend a result of Popken concerning the numerators of the Taylor coefficients of algebraic functions and combine it with a result of Mahler on lacunary power series to prove an extension of a special case of a result of Cohn on the transcendence of functional values of lacunary power series evaluated at rational points.
\end{abstract}

1. Introduction. In 1959 Popken [4] proved the following result [4, Theorems 1, 2, p. 203] for numerators of Taylor coefficients of algebraic functions:

POPKEN'S THEOREM. Let the power series $f(z)=\sum_{n=0}^{\infty} a_{n} z^{n}$, with rational coefficients $a_{n}(n=0,1,2, \ldots)$ and convergent in a neighbourhood $|z|<R$ of the origin, represent a branch of an algebraic function which is not a polynomial. Let $b$ denote $a$ rational number such that $0<|b|<R$. Let $S_{n}=\sum_{\nu=0}^{n} a_{\nu} b^{\nu}(n=$ $0,1,2, \ldots)$. Denote by $p_{n}$ the largest prime divisor in the numerator of $S_{n}$.

(i) If $f(b) \neq 0$, then $\lim \sup _{n \rightarrow \infty} p_{n}=\infty$.

(ii) If $f(b)$ is an irrational number, then $\lim _{n \rightarrow \infty} p_{n}=\infty$. (This last statement implies that the limit exists in an extended sense.)

By studying Popken's proof of this theorem, one sees that the condition that $f(z)$ is an algebraic function can be somewhat modified without affecting the proof. We illustrate this remark by using Popken's original proof, but with different hypotheses, to derive a similar result. This new version of Popken's theorem, combined with a result of Mahler [3, Theorem 1, p. 57], enables us to obtain an interesting consequence about transcendental values of lacunary analytic functions taken at rational points. This last result is an extension of a particular case of the following theorem due to Cohn [2].

COHN'S THEOREM. Let $f(z)=\sum_{k=0}^{\infty} a_{k} z^{e_{k}}$ be a lacunary power series with rational coefficients $a_{k}=p_{k} / q_{k}(k \geq 0)$. Let $R$ be the radius of convergence of $f$, $A_{k}=\max _{i=0, \ldots, k}\left|a_{i}\right|$, and $M_{k}$ the least common multiple of $q_{0}, \ldots, q_{k}$. If

$$
\lim _{k \rightarrow \infty} \frac{e_{k}+\log M_{k}+\log A_{k}}{e_{k+1}}=0
$$

then $f(b)$ is transcendental for every algebraic number $b$, with $0<|b|<R$.

This formulation of Cohn's result is taken from Cijsouw and Tijdeman [1]; indeed, Cijsouw and Tijdeman generalized Cohn's result to the case where the $a_{k}$ are algebraic integers.

Received by the editors April 12, 1984.

1980 Mathematics Subject Classification. Primary 10F35, 30B10. 


\section{A new version of Popken's Theorem.}

THEOREM 1. Let $f(z)=\sum_{n=0}^{\infty} a_{n} z^{n}$ be a power series which is not a polynomial with rational integral coefficients $a_{n}(n=0,1,2, \ldots)$ converging in a neighbourhood $|z|<R$ of the origin. Let $b$ denote a rational number such that $0<|b|<R$. Let $S_{n}=\sum_{\nu=0}^{n} a_{\nu} b^{\nu}(n=0,1.2, \ldots)$. Let $p_{n}$ denote the largest prime divisor in the numerator of $S_{n}$.

(i) If $f(b)$ is a nonzero algebraic number, then $\limsup _{n \rightarrow \infty} p_{n}=\infty$.

(ii) If $f(b)$ is an algebraic irrational number, then $\lim _{n \rightarrow \infty} p_{n}=\infty$.

PROOF. (i) Put $b=u / v$, where $u$ and $v>0$ are rational integers. Then

$$
S_{n}=\sum_{\nu=0}^{n} \frac{a_{\nu} u^{\nu}}{v^{\nu}}=\frac{x_{n}}{y_{n}}
$$

with $y_{n}=v^{n}$ and $x_{n}$ an integer for $n \geq 1$. Denote the prime divisors of $v$ by $p_{1}, p_{2}, \ldots, p_{g}$. Now suppose the assertion $\lim \sup _{n \rightarrow \infty} p_{n}=\infty$ is false. Then all integers $y_{i}, x_{i}$ have a finite number of prime divisors $p_{1}, p_{2}, \ldots, p_{w}(w \geq g)$. Thus

$$
x_{i}= \pm p_{1}^{\xi_{1}} p_{2}^{\xi_{2}} \cdots p_{w}^{\xi_{w}}, \quad y_{i}=p_{1}^{\eta_{1}} p_{2}^{\eta_{2}} \cdots p_{2}^{\eta_{w}} \quad(i=0,1,2, \ldots),
$$

where the $\xi$ 's and $\eta$ 's are nonnegative integers.

Since $0<|b|<R$, there exists a positive number $\delta$ so small that $\omega:=$ $(\delta+1 / R)|b|<1$. If $R^{\prime}(\geq R)$ denotes the radius of convergence of $\sum a_{n} z^{n}$, then

$$
\limsup _{n \rightarrow \infty} \sqrt[n]{\left|a_{n}\right|}=\frac{1}{R^{\prime}}
$$

Hence, for sufficiently large $i$,

$$
\left|f(b)-\frac{x_{i}}{y_{i}}\right|=\left|\sum_{\nu=i+1}^{\infty} a_{\nu} b^{\nu}\right| \leq \sum_{\nu=i+1}^{\infty}\left(\delta+\frac{1}{R}\right)^{\nu}|b|^{\nu}=\frac{\omega^{1+i}}{1-\omega} .
$$

Choose a positive number $k$ so small that $1 / v^{k}>\omega$. Then for sufficiently large $i$,

$$
y_{i}^{-k}=v^{-k i}>\omega^{i} /(1-\omega) \text {. }
$$

Hence, for sufficiently large $i$,

$$
\left|f(b)-x_{i} / y_{i}\right|<\omega^{i} /(1-\omega)<y_{i}^{-k} .
$$

Since $f(b) \neq 0$ is an algebraic number, by Ridout's theorem [5],

$$
f(b)=x_{i} / y_{i} \quad \text { for sufficiently large } i \text {. }
$$

It follows that $a_{i} b^{i}=S_{i}-S_{i-1}=0$. Hence, $a_{i}=0$ for sufficiently large $i$, so $f(z)$ is necessarily a polynomial, which is a contradiction.

(ii) The proof is similar to (i). We assume the assertion is false. Then there exists an increasing sequence $\left(n_{j}\right)$ such that all numerators of $S_{n_{j}}$ can be formed from a finite number of primes. By the same arguments as in (i), using $\left(n_{j}\right)$ instead of $(n)$ and $S_{n_{j}}$ rather than $S_{n}$, we arrive at the fact that, for sufficiently large $j$, $f(b)=x_{n_{j}} / y_{n_{j}}$. This contradicts the irrationality of $f(b)$, and Theorem 1 is proved.

We remark that, with only a slight change in the proof, Theorem 1 is still true if the coefficients $a_{k}$ satisfy the Eisenstein condition, i.e. $\exists N \in \mathbf{N}$ such that $N^{k} a_{k} \in \mathbf{Z}$ $(k=0,1,2, \ldots)$. 
3. Transcendental values of lacunary power series. For our application we need the following result of Mahler [3, Theorem 1, p. 57].

MAHLER'S THEOREM. Let $f(z)=\sum_{n=0}^{\infty} a_{n} z^{n}$ be a power series with rational integral coefficients converging in a neighbourhood $|z|<R$ of the origin. Suppose $f(z)$ satisfies the gap condition, that is, there are two infinite sequences of integers, $\left\{r_{n}\right\}$ and $\left\{s_{n}\right\}$, satisfying

$$
0=s_{0} \leq r_{1}<s_{1} \leq r_{2}<s_{2} \leq r_{3}<s_{3} \leq \cdots, \quad \lim _{n \rightarrow \infty} \frac{s_{n}}{r_{n}}=\infty,
$$

such that $a_{h}=0$ if $r_{n}<h<s_{n}$, but $a_{r_{n}} \neq 0, a_{s_{n}} \neq 0(n=1,2,3, \ldots)$. Let $b$ be an algebraic number satisfying $|b|<R$. Then $f(b)$ is algebraic if and only if there exists a positive integer $N=N(b)$ such that $P_{n}(b)=0$ for all $n \geq N$, where

$$
P_{n}(z)=\sum_{h=s_{n}}^{r_{n+1}} a_{h} z^{h} \quad(n=0,1,2, \ldots) .
$$

We are now ready to prove

THEOREM 2. Let $f(z)=\sum_{n=0}^{\infty} a_{n} z^{n}$ be a lacunary power series with properties as in Mahler's Theorem. If $b$ is a rational number satisfying $0<|b|<R$, then $f(b)$ is either a rational or a transcendental number.

Proof. Suppose, to the contrary, that $f(b)$ is an algebraic irrational number. Then by Mahler's Theorem there exist a positive integer $N=N(b)$ and an increasing sequence of positive integers $\left(n_{j}\right)$ such that $S_{n_{j}}=S_{r_{N}}$ for all $j$. Thus the limiting value of the largest prime divisors $p_{n}$ of $S_{n}$ (as $n$ tends to infinity) either does not exist, or, if it does, it is never infinite. This contradicts Theorem 1(ii), and our result follows.

We conclude with a few remarks.

(1) The case where $s_{n}=r_{n+1}(n=0,1,2, \ldots)$ in Theorem 2 corresponds to a special case of Cohn's Theorem mentioned earlier. In this case $f(b)$ is necessarily a transcendental number, because, if not, Mahler's Theorem then implies $P_{n}(b)=$ $a_{s_{n}} b^{s_{n}}=0$ for all sufficiently large $n$, so $f(z)$ reduces to a polynomial, which is a contradiction.

(2) The possibility, in Theorem 2, of $f(b)$ being a rational number does indeed exist, as shown by the following example. Take for $n \geq 1$,

$$
r_{n+1}=s_{n}+1, \quad a_{r_{n+1}} / a_{s_{n}}=a_{s_{n}+1} / a_{s_{n}}=-1 / b .
$$

Then $P_{n}(b)=0$ for all $n \geq 1$, so $f(b)=a_{0}+a_{r_{1}} b^{r_{1}}$ is a rational number.

\section{REFERENCES}

1. P. L. Cijsouw and R. Tijdeman, On the transcendence of certain power series of algebraic numbers, Acta Arith. 23 (1973), 301-305.

2. H. Cohn, Note on almost-algebraic numbers, Bull. Amer. Math. Soc. 52 (1946), 1042-1045.

3. K. Mahler, Arithmetic properties of lacunary power series with integral coefficients, J. Austral. Math. Soc. 5 (1965), 55-64.

4. J. Popken, Arithmetical properties of the Taylor-coefficients of algebraic functions, Proc. Kon. Ned. Akad. Wetensch. 62 (1959), 202-210.

5. D. Ridout, Rational approximations to algebraic numbers, Mathematika 4 (1957), 125-131.

Department of Mathematics, University of Texas, Austin, Texas 78712

Current address: Department of Mathematics, Kasetsart University, Bangkok 10900, Thailand 